\title{
DECLARACIÓN DE INADMISIBILIDAD FRENTE A UN SEGUNDO REQUERIMIENTO EN LA SEGUNDA GESTIÓN PENDIENTE. LA JURISPRUDENCIA DEL TRIBUNAL CONSTITUCIONAL*
}

\section{Francisco CaŃas SEPúlveda**}

RESUMEN: En el presente trabajo se describe lo que el Tribunal Constitucional chileno dictamina frente a un requerimiento de inaplicabilidad por inconstitucionalidad presentado por segunda vez. De esta descripción es posible concluir que nuestro Tribunal declara en la mayoría de los casos inadmisible el requerimiento presentado por segunda vez, en la misma gestión pendiente.

PALABRAS CLAVE: Admisibilidad, Tribunal Constitucional, Gestión pendiente

\section{DECLARATION OF INADMISSIBILITY AGAINST A SECOND PETITION ABOUT THE SAME PENDING PROCEDURE. THE JURISPRUDENCE OF THE CONSTITUTIONAL COURT}

ABSTRACT: This paper describes what the Chilean Constitutional Court dictates against a petition of unconstitutionality resubmitted. From this description it is possible to conclude that our Court declares in most of the cases inadmissible the petition submitted by a second time about the same pending procedure. dure.

KEY WORDS: Admissibility, Constitutional Court, pending proce-

Fecha de recepción: 19 de junio de 2012.

Fecha de aprobación: 26 de abril de 2013

* Candidato a Magíster en Derecho Público de la Universidad Diego Portales y Ayudante del Departamento de Derecho Público de la misma Universidad. e-mail: f.canas.sepulveda@ gmail.com

Agradezco a los profesores Rodolfo Figueroa, Emilio Pffefer y Jorge Correa Sutil por sus comentarios que me fueron de mucha utilidad. No obstante, todos los errores e imprecisiones son de mi entera responsabilidad. 
Sumario: 1) Introducción. 2) Las normas de la ley orgánica constitucional del Tribunal Constitucional. 3) Las normas de la ley orgánica constitucional del Tribunal Constitucional. 4) Las normas de la ley orgánica constitucional del Tribunal Constitucional. 5) Bibliografía.

\section{1) INTRODUCCIÓN}

Desde la reforma constitucional del año 2005, nuestro Tribunal Constitucional detenta el control constitucional de la legislación. Para ejercerlo, se han configurado nuevos procedimientos que propenden a que el Tribunal Constitucional pueda conocer de una serie de conflictos constitucionales, siendo uno de ellos el de la inaplicabilidad ${ }^{1}$. Luego de un gran tiempo sin que el legislador configurara un proceso para esta acción, el 2009 dictó la Ley 20.381 que modifica la Ley Orgánica del Tribunal Constitucional ${ }^{2}$. En esta ley se previó, para la acción de inaplicabilidad, un proceso ${ }^{3}$ que consta de tres etapas ${ }^{4}$. La primera es la de "acogimiento a trámite", la segunda es la de "admisibilidad" y la tercera etapa es la de "fondo del asunto". En las etapas de "acogimiento a trámite" y "admisibilidad" se prevé la posibilidad de que el recurso sea desechado por no cumplir con alguno de los requisitos establecidos en la ley. En efecto, en la LOCTC señala que si el requerimiento no cumple con los requisitos legales, este "se tendrá por no presentado". ¿Eso implica que el requerimiento que fue declarado inadmisible no se puede volver a presentar? En este trabajo intentaremos responder a esa pregunta desde una perspectiva jurisprudencial.

1 Sobre la acción de inaplicabilidad se han escrito una gran cantidad de trabajos. Por ejemplo, según el Catálogo de Publicaciones del Anuario de Derecho Público de la Universidad Diego Portales, solo el año 2010 se publicaron al menos 17 artículos académicos y por lo menos un libro, acerca de la acción de inaplicabilidad. En el año 2011 esta cifra ascendió dos libros. Llama la atención que, según el Anuario de Derecho Público UDP en 2011, no se registren artículos sobre la acción -y el procedimiento- de inaplicabilidad. Sobre la historia de la acción de inaplicabilidad, véase Navarro, Enrique (2010). "Acción de inaplicabilidad por inconstitucionalidad. Notas sobre la evolución histórica en Chile". Revista Actualidad Jurídica, No 22.

2 Un comentario detallado de las modificaciones a la LOCTC puede verse en CEA, José Luis (2010). "Nueva Ley Orgánica del Tribunal Constitucional: Una síntesis compleja”. Revista Chilena de Derecho, Vol. 37, No 1.

3 Una explicación del procedimiento de inaplicabilidad puede verse en PICA, Rodrigo (2010). "El procedimiento correspondiente al proceso de inaplicabilidad por inconstitucionalidad en la Ley Orgánica Constitucional del Tribunal Constitucional”. En Nogueira, Humberto (editor): Temas de derecho procesal constitucional: Reflexiones jurídicas sobre competencias del Tribunal Constitucional y la nueva LOC del Tribunal Constitucional. Santiago de Chile: Librotecnia.

4 Un estudio detallado de la jurisprudencia del Tribunal Constitucional sobre la acción de inaplicabilidad puede encontrarse en CORREA, Jorge (2011) Inaplicabilidad por inconstitucionalidad en la jurisprudencial del Tribunal Constitucional. Santiago de Chile: Abeledo Perrot. 
El Tribunal Constitucional declara, en la mayoría de los casos, inadmisible el requerimiento presentado, en una misma gestión pendiente, por segunda vez ${ }^{5}$. Entre el 2005 y el 2012 el Tribunal ha tenido la oportunidad de referirse a esta materia en catorce casos. En doce de estos casos el Tribunal Constitucional declaró inadmisible el requerimiento. En seis de ellos fueron declarados inadmisibles por presentarse en una segunda oportunidad. En los otros seis el Tribunal declaró inadmisible el requerimiento por otra causal, pero arguyó como una razón a sumar el que el requerimiento ya se había presentado anteriormente. En dos casos el Tribunal declaró admisible el requerimiento. Solo en uno se discutió acerca del fondo del requerimiento ${ }^{6}$. El presente trabajo tiene por objeto describir y analizar esa jurisprudencia, y para hacerlo, se identificarán brevemente las normas de la Ley Orgánica del Tribunal Constitucional sobre esta materia, se describirán los casos y luego realizaré una breve conclusión.

La importancia de este estudio es doble: en primer lugar, permite al litigante, ante el Tribunal Constitucional, conocer la jurisprudencia del Tribunal Constitucional de manera de diseñar su estrategia para el caso; en segundo término, solo conociendo la jurisprudencia del Tribunal Constitucional, la academia podrá evaluar si lo que ha venido haciendo el Tribunal en este asunto es correcto o si por el contrario es necesario realizar algunas modificaciones o plantear nuevos elementos en esta discusión.

\section{2) LaS NORMAS DE LA LEY ORGÁNICA CONSTITUCiONAL DEL TribU- NAL CONSTITUCIONAL}

La Ley Orgánica del Tribunal Constitucional (en adelante la LOCTC) prescribe los requisitos con los que debe cumplir el requerimiento de inaplicabilidad para ser acogido a tramitación, los que deben revisarse en el examen de admisibilidad ${ }^{7}$. La LOCTC distingue ${ }^{8}$ dos clases de requisitos, los requisitos formales y los otros requisitos ${ }^{9}$. La LOCTC señala lo que sucede si no se cumple con los requisitos estableci-

5 Dentro de esta situación existen varias hipótesis fácticas. Se me ha sugerido distinguir cada hipótesis para así examinar, en cada caso, lo que ha señalado el Tribunal Constitucional. Sin embargo, como se mostrará en el desarrollo de este trabajo, solo algunas distinciones son útiles, pues el Tribunal Constitucional pareciera no distinguir en todos los casos. Tampoco es útil distinguir cuando el TC no distingue, pues este trabajo solo tiene pretensiones descriptivas, y por tanto, no se pretende señalar lo que el Tribunal debe hacer.

6 Toda esta información se puede ver en el cuadro № 2.

7 Artículo 82 inciso 1.

8 La LOCTC distingue entre ambos requisitos, pero no señala cuáles son los unos y los otros. Esto tiene un efecto crítico ya que, según la ley, los requisitos formales son subsanables dentro de tercero día. Si el requirente no sabe cuáles son los requisitos formales no sabrá si puede subsanar el requerimiento. Artículo 82 inciso 2. 
dos en los artículos 79 y 80: el requerimiento se tendrá por no presentado. Esta es la regla general. No obstante -seńala la LOCTC- tratándose de defectos de forma, el requirente cuenta con un plazo de tres días para subsanar. Luego, si pasado los tres días, el requirente no subsana los defectos formales, el requerimiento "se tendrá por no presentado".

Siguiendo la lógica de la LOCTC, si el requerimiento se tiene por no presentado, se puede volver a presentar. ¿Es posible volver a presentar, una vez declarado inadmisible, el requerimiento de inaplicabilidad? El profesor Tavolari, analizando la Ley Orgánica del Tribunal Constitucional ha señalado que " $[\mathrm{e}] \mathrm{n}$ todos los casos en que el requerimiento, por ser declarado inadmisible, se tenga por no presentado, para todos los efectos legales, no puede caber duda alguna de que se puede renovar" ${ }^{10}$, es decir, se puede volver a presentar un requerimiento habiendo subsanado los defectos formales, en la misma gestión pendiente. En esa línea el profesor Nogueira, antes de la reforma a la LOCTC, señalaba que: "la presentación de un incidente de inconstitucionalidad por vicios de forma no obsta para presentar luego un incidente de inconstitucionalidad en virtud de un vicio de inconstitucionalidad de fondo" 11.

En la legislación civil se prescribe similar efecto a la demanda que fuese retirada por el demandante, antes de que fuese notificada ${ }^{12}$. En esos casos, los autores han señalado que el demandante queda "en plena libertad para presentarla posteriormente ante el mismo u otro tribunal"13. La analogía que planteó tiene una base gramatical pues el artículo 148 del Código de Procedimiento Civil señala que la demanda "se considerará como no presentada”. El lenguaje del legislador es casi idéntico ${ }^{14}$.

¿Existe alguna norma que prohíba que se pueda presentar por segunda vez un requerimiento en la misma gestión pendiente?

10 Tavolari, Raúl (2010). "La cosa juzgada en el control de constitucionalidad”. Revista de derecho público, Vol. 72, p. 479.

11 Nogueira, Humberto (2005). "El control represivo concreto y abstracto de inconstitucionalidad”. En Zúñiga, Francisco (editor) Reforma Constitucional. Santiago: Editorial LexisNexis, p. 597.

12 El artículo 148 del Código de Procedimiento Civil señala que: "Antes de notificada una demanda al demandado, podrá el actor retirarla sin trámite alguno, y se considerará como no presentada". Lo destacado es mío.

13 Otero, Miguel (2000) Derecho Procesal civil. Modificaciones a la legislación (1988-2000). Santiago de Chile: Editorial Jurídica de Chile, p. 124. En el mismo sentido Colombo, Juan (2008) Los actos procesales. Santiago de Chile: Editorial Jurídica de Chile, p. 90; Casarino, Mario (2005) Manual de derecho procesal. Santiago de Chile: Editorial Jurídica de Chile, p.176.

14 Cabe hacer notar que, al igual que en la legislación civil, el requerimiento en esta etapa aun no es notificado al requirente ni al Tribunal de la gestión pendiente, lo que reafirma la analogía. Además, el requirente antes de la notificación puede retirar el requerimiento. Véase el artículo 86 de la LOCTC. 
A este respecto resulta necesario analizar el artículo 90 de la LOCTC, porque se trata de una prohibición que podría aplicar a esta materia. El artículo señala:

"Artículo 90. Resuelta la cuestión de inaplicabilidad por el Tribunal Constitucional, no podrá ser intentada nuevamente, por el mismo vicio, en las sucesivas instancias o grados de la gestión en que se hubiere promovido".

La citada disposición señala una prohibición aplicable a un requerimiento de inaplicabilidad resuelto, es decir, a uno en que se haya discutido acerca del fondo del asunto. Además, dicha prohibición no es absoluta porque seńala que el requerimiento de inaplicabilidad no puede ser intentado nuevamente por el mismo vicio, lo que implica que se puede volver a presentar por un vicio distinto. Esto es importante porque al parecer va contra la lógica de la LOCTC que se prohíba la presentación de un segundo requerimiento de inaplicabilidad, en la misma gestión pendiente, si ni siquiera en el caso en que dicho requerimiento se haya resuelto la prohibición es total. Como dice el proverbio jurídico: "quien puede lo más, puede lo menos".

De esta manera, pareciera que la LOCTC no prohíbe que, ante un requerimiento declarado inadmisible, se pueda volver a presentar otro. Para corroborar si el Tribunal Constitucional ha entendido el texto así, examinaremos la jurisprudencia sobre este punto

\section{3) AnÁlisis de la Jurisprudencia del Tribunal Constitucio- NAL RESPECTO DEL REQUERIMIENTO PRESENTADO POR SEGUNDA VEZ EN LA MISMA GESTIÓN PENDIENTE}

Desde el 2005 hasta el 2012, se han presentado por segunda vez en la misma gestión pendiente catorce requerimientos, tal como se aprecia en el cuadro 1 .

\section{CuAdro $1^{15}$}

$\begin{array}{cc}\text { Rol } & \text { Primera presentación } \\ 1 & 1265-2008 \\ 2 & 1420-2009 \\ 3 & 1036-2008 \\ 4 & 1581-2010 \\ 5 & 1590-2010\end{array}$

Segunda presentación

1282-2008

1442-2009

1311-2009

$1671-2010$

$1672-2010$

15 Cuadro de elaboración propia. 


Rol
6
7
8
9
10
11
12
13
14

Primera presentación
$1840-2010$
$1678-2010$
$1977-2011$
$2309-2012,2310-2012$
$1937-2011$
$1938-2011$
$1936-2011$
$1739-2010$
$2218-2012$

Segunda presentación
$1881-2010$
$1834-2010$
$2013-2011$
$2331-2012$
$2088-2011$
$2089-2011$
$2090-2011$
$1759-2010$
$2247-2012$

Si desglosamos esta información nos encontraremos con que solo dos ${ }^{16}$ requerimientos pasaron las etapas de "acogimiento a trámite" y "admisibilidad". Los otros doce restantes fueron declarados inadmisibles. De esos diez requerimientos, seis ${ }^{17}$ fueron declarados inadmisibles utilizando tres fórmulas que tiene por objetivo objetar la presentación de un segundo requerimiento. Otros seis casos fueron declarados inadmisibles por otra causal ${ }^{18}$. El desglose de esta información se puede apreciar en el cuadro № 2 .

\section{CUADro $2^{19}$}

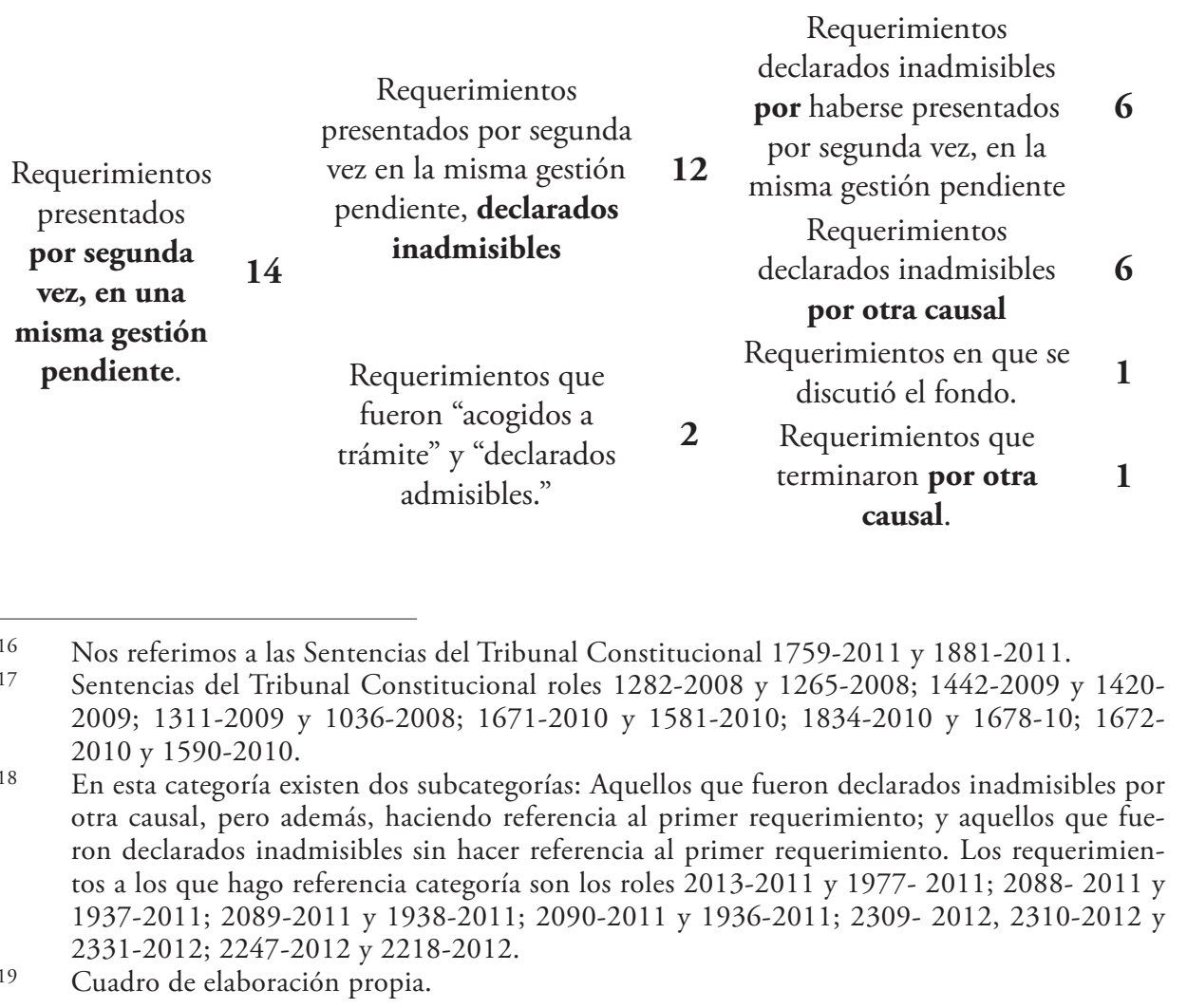


A continuación examinaré tres fórmulas que el Tribunal Constitucional ha utilizado para declarar inadmisible el segundo requerimiento, por haberse presentado uno anteriormente.

a. En primer lugar, el Tribunal Constitucional, ha manifestado que el segundo requerimiento en los hechos sería un recurso en contra de la sentencia interlocutoria que declaró inadmisible el primer requerimiento. El Tribunal Constitucional ha señalado:

"Que, efectuado el examen del requerimiento deducido, se advierte que este importa, en el becho, un recurso que intenta dejar sin efecto la sentencia interlocutoria dictada el 9 de julio del año en curso, que declaró inadmisible la misma acción de inaplicabilidad por inconstitucionalidad presentada ante esta Magistratura Constitucional"20.

El Tribunal estima que con la segunda presentación se vulneraría el artículo 84 inciso final de la LOCTC. Esta disposición señala que:

"La resolución que declare la admisibilidad o inadmisibilidad del requerimiento no será susceptible de recurso alguno”.

Lo señalado por el Tribunal no deja del todo claro por qué el segundo requerimiento sería un recurso. Tampoco queda claro si es que verdaderamente contra la resolución no procede recurso alguno, pues el TC en un caso pareciera exigirle al requirente un requerimiento que haga las veces de recurso. El Tribunal seńala que:

"el requirente planteó el mismo conflicto que hoy vuelve a someter a esta Magistratura (...) el actor no bace referencia alguna, en su nueva presentación, acerca de los motivos de la diferencia entre el estado actual de la causa en que incide la acción deducida (...) como tampoco al contenido de esta última resolución y la razón por la cual, a su juicio, no obstante ello, es procedente presentar este nuevo requerimiento" 21 .

20 Tribunal Constitucional. 18 de diciembre de 2008. Rol 1282-08. "Requerimiento de inaplicabilidad de Sonia Angélica Cifuentes Olea respecto del artículo 38 ter de la Ley $\mathrm{N}^{\circ}$ 18.933, en la causa sobre recurso de protección Rol de Ingreso $\mathrm{N}^{\circ} 8798-2008$, de la Corte de Apelaciones de Santiago". Considerando 4. Lo destacado es mío.

21 Tribunal Constitucional. 26 de octubre de 2010. Rol 1834-10. "Requerimiento de inaplicabilidad por inconstitucionalidad presentado por Alberto Michel Haddad Valech respecto del artículo 212 del Código Civil, en los autos Rol No 2642-2009 sobre recurso de apelación interpuesto ante la Corte de Apelaciones de Santiago en contra de la sentencia del Primer Juzgado de Familia de Santiago". Considerandos 6 y 7. Lo destacado es mío. 
b. En segundo lugar el Tribunal Constitucional ha señalado que la segunda presentación sería un intento por burlar la ley (en particular el artículo 84 inciso final) estimándolo, por esta razón, carente de fundamento razonable, declarándolo inadmisible. En efecto el Tribunal Constitucional señaló:

"El tribunal concluyó que "no cabe deducir recurso alguno en contra de las resoluciones que dicte la Sala en ejercicio de la atribución que se le asigna en la materia, el intento del actor por burlarla, a través de la interposición de un requerimiento de inaplicabilidad que solo es nuevo en apariencia, produce el efecto de estimar que la acción deducida en estos autos no contiene una impugnación razonablemente fundada $y$, por consiguiente, debe ser declarada inadmisible"22.

c. En tercer lugar, el Tribunal Constitucional ha señalado que con la primera presentación el derecho del requirente precluye, no pudiendo presentar otro requerimiento en la misma gestión pendiente. El tribunal estimó que:

"debe concluirse que en el proceso que constituye la gestión pendiente este es el segundo requerimiento de inaplicabilidad por inconstitucionalidad interpuesto por la misma materia y fundamento, por lo cual debe ser desestimado, toda vez que al haber consumado en el requerimiento anterior su derecho, este precluyó" 23.

El Tribunal reafirmando esta fórmula ha señalado que se debe:

"plantear todas las infracciones constitucionales en una misma y sola oportunidad, como única forma de producir certeza en los derechos que invoca y de obtener de una vez el pronunciamiento del Tribunal sobre los mismos. El requirente tiene la facultad de ba-

22 Tribunal Constitucional. 21 de abril de 2010. Rol 1671-10. "Requerimiento de inaplicabilidad por inconstitucionalidad de Pedro Hernán Loch Contreras, respecto de los artículos 1.545 del Código Civil, 38 de la Ley $\mathrm{N}^{\circ} 18.933$ y $2^{\circ}$ de la Ley $\mathrm{N}^{\circ} 20.015$, en los autos Rol N ${ }^{\circ}$ 1091-2009 sobre recurso de protección interpuesto ante la Corte de Apelaciones de Santiago en contra de la Isapre Vida Tres S.A.”. Considerandos $7^{\circ}$ y 8.

23 Tribunal Constitucional. 2 de abril de 2009. Rol 1311-09. "Requerimiento de inaplicabilidad por inconstitucionalidad de Mario Alberto Cortés Alarcón respecto de los artículos 103 y siguientes, en especial del actual 138, antes 135, del Decreto con Fuerza de Ley No 3, del año 1997, que fija el texto refundido y sistematizado de la Ley General de Bancos, en la causa Rol 4057-1999, del Vigésimo Juzgado Civil de Santiago, caratulada "Banco Santander con Cortés Alarcón, Mario Alberto”, actualmente en la Corte de Apelaciones de Santiago, Rol No C-9095-2001”. Considerando 15. Lo destacado es mío. 


\section{cer uso de este derecho, pero debe hacerlo en el contexto de lo que exige un proceso racional y justo" 24 .}

El Tribunal en un fallo reciente ha sido más enfático aún, cerrando todas las puertas a una segunda presentación en la misma gestión pendiente. En ese fallo razonó así:

\section{"la resolución que declara la inadmisibilidad del requerimien- to de inaplicabilidad, al impedir la prosecución del proceso, es una sentencia interlocutoria y como tal, produce efecto de cosa juzgada"25.}

Como se puede apreciar el Tribunal ha utilizado tres fórmulas para declarar inadmisible el requerimiento en la segunda presentación. Sin embargo, esta declaración de inadmisibilidad no ha sido unánime en un caso. En efecto, en el fallo rol 1311-2009 el Ministro Fernández no estuvo de acuerdo con la mayoría y señaló que el requerimiento declarado inadmisible puede, luego de ser enmendado, presentarse nuevamente. Señaló que:

"si la declaración de inaplicabilidad del requerimiento se sustentara en la falta de razonabilidad de sus fundamentos, aquel enmendado puede ser presentado nuevamente, aun cuando se admita haber precluido la facultad procesal que ya habia sido ejecutada válidamente en su primera presentación. Lo dicho, en la medida en que esta Magistratura no haya emitido pronunciamiento jurisprudencial sobre la peculiar entidad de la cosa juzgada en materia constitucional"26.

Debe notarse que el Ministro Fernández pareciera concordar con la mayoría respecto de que en este tipo de casos sí existe preclusión, pero no

24 TC, Rol 1311-09, Considerando 9. Es relevante que el Tribunal Constitucional señale que lo anterior lo exige el justo y racional procedimiento, pues como se mostrará en la última parte de este trabajo, este efecto produce un resultado contradictorio con las demás normas (irracional) y desproporcionado (injusto).

25 Tribunal Constitucional. 28 de junio de 2012. Rol 2247-2012. "Requerimiento de inaplicabilidad por inconstitucionalidad presentado por Gastón Holzapfel Gross respecto de los artículos 3, 229, 334, 342, letra f), 370 y 483 del Código Procesal Penal, en los autos sobre reforma procesal penal, RIT 1930-2004, RUC 0410014473-0, del Juzgado de Garantía de Valdivia, de que conoce actualmente la Corte Suprema, por recurso de nulidad, bajo el Rol N³894-2012". Considerando 9.

26 Prevención del Ministro Fernández en el fallo rol 1311-2009. Debe ponerse en relieve una de las ideas que en este párrafo seńala Fernández: no existe un pronunciamiento sobre la cosa juzgada constitucional. Esto porque pareciera indicar que, tal como lo señalamos anteriormente, no existe una norma que prohíba la segunda inaplicabilidad, de lo contrario, el pronunciamiento existiría. Lo destacado es mío. 
estima que esta sea relevante. Otra idea importante es que Fernández cree que el requerimiento enmendado sí se puede presentar nuevamente (en concordancia con el artículo 90 de la LOCTC $^{27}$ ). La mayoría explícitamente niega esta posibilidad señalando que:

\section{"la distinta formulación que se presenta en el texto de la acción bajo examen, sobre las consideraciones de derecho en las que ella se apoya, no cambia la circunstancia de tratarse de la misma pre- tensión ya declarada inadmisible"28.}

Como se dijo anteriormente, seis de los casos revisados fueron declarados inadmisibles por el Tribunal Constitucional señalando como una causal de inadmisibilidad el haberse presentado un requerimiento con anterioridad en la misma gestión pendiente ${ }^{29}$. Para hacerlo ha utilizado tres fórmulas: que el segundo requerimiento en los hechos sería un recurso; que el segundo requerimiento implicaría una burla a la ley; que la presentación del primer requerimiento precluiría la posibilidad de presentar un segundo requerimiento.

Las hipótesis fácticas en las que el Tribunal Constitucional declara la inadmisibilidad, ante un segundo requerimiento, son: i) el requerimiento es presentado en la misma gestión pendiente, con el mismo fundamento normativo y por la misma parte que el primero; ii) el requerimiento es presentado en la misma gestión pendiente, por la misma parte pero con distinto fundamento normativo; iii) el requerimiento es presentado en la misma gestión pendiente, por el mismo requirente, con el mismo fundamento normativo, pero subsanando los vicios formales por los cuales el TC tuvo por no presentado el primero. El punto decisivo-entonces- será la gestión pendiente ${ }^{30}$. De la anterior revisión de fallos es posible extraer -además- que la fórmula de la preclusión no ha sido unánime en un caso y, por último se mostró que la mayoría sostiene que la distinta formulación del requerimiento no es relevante para su admisibilidad.

Esto, a pesar de que al momento de dictarse el fallo en este caso, la LOCTC aún no había sido reformada, de manera que el ministro Fernández no tuvo en ese momento un fundamento normativo del cual aferrarse. Al parecer, en la nueva LOCTC se reparó en el asunto, aunque como hemos visto, en la mayoría de los casos el TC sigue declarando inadmisible los requerimientos a pesar de que se puedan volver a presentar enmendados.

Tribunal Constitucional. 5 de agosto de 2009. Rol 1442-2009. "Requerimiento de inaplicabilidad por inconstitucionalidad de Isapre MASVIDA S.A. respecto del inciso tercero del artículo transitorio de la Ley No 20.317, en causa Rol de ingreso No 2276-2009 de la Corte de Apelaciones de Santiago". Considerando 5. Lo destacado es mío. A pesar de que esa causal no existe en la LOCTC.

30 Si la gestión pendiente es distinta, el Tribunal declarará admisible el requerimiento. Esto se puede apreciar con mucha claridad en los casos 1960 y 1961. En estos casos, los requerimientos eran exactamente iguales y se presentaron el mismo día y a la misma hora. La única diferencia era la gestión pendiente. 
Ahora corresponde ver los otros los casos restantes.

\section{Casos declarados inadmisibles por otra causal}

En esta parte parece conveniente distinguir dos tipos de casos. Por un lado, aquellos casos en que se declaró inadmisible el segundo requerimiento haciendo presente - de forma expresa- la objeción por la segunda presentación, y por el otro, aquellos casos en que el TC no se pronuncia sobre esa objeción y lo declara inadmisible por otras causales. Entre los primeros, durante el periodo de estudio, hemos detectado 5 casos. Existe uno, en que el TC no se pronunció -en la segunda presentación- acerca de la primera, y declaró inadmisible por otra causal. Describiremos ambas situaciones, pero partiremos por la primera descrita:

Existen tres ${ }^{31}$ casos presentados por el mismo abogado, solicitando que se inaplique los artículos $2^{\circ}$ y $3^{\circ}$ del Decreto Ley 3500 . El punto que genera dudas es que el abogado en sus primeras presentaciones (o requerimientos) señaló la gestión pendiente y sus inaplicabilidades fueron declaradas inadmisibles "derechamente" 32 . Luego, en su segunda presentación, el abogado no invocó la gestión pendiente en la que pretendía inaplicar el precepto $^{33}$ y el Tribunal lo declaró inadmisible por no haberlo hecho ${ }^{34}$.

Lo interesante de estos casos es que al final de las sentencias el Tribunal Constitucional señaló que "el mismo requirente impugno las mismas normas" en otro requerimiento que ya había sido declarado inadmisible.

Por ejemplo, en el caso 2088-2011 señaló:

"Que, a mayor abundamiento, el mismo requirente impugno las mismas normas en el proceso Rol N¹937-11-INA, libelo que fue declarado inadmisible con fecha 30 de marzo de 2011 " 35 .

31 Los casos a los que me refiero son: Roles $1937-2011$ y 2088-2011; 1938-2011 y 20892011; 1936-2011 y 2090-2011.

32 Fueron declarados inadmisibles porque el requirente no expuso el conflicto constitucional y tampoco argumentó cómo el precepto invocado produciría una infracción constitucional, de manera que el requerimiento no cumple con la exigencia de encontrarse "razonablemente fundado". El Tribunal señaló además que el certificado de gestión pendiente no contenía los elementos exigidos en la ley

33 Esta situación pudo haber sucedido por inadvertencia o por una estrategia procesal. Si fue por la segunda razón, es probable que el abogado haya conocido la jurisprudencia del TC acerca de los segundos requerimientos en la misma gestión pendiente.

$34 \quad C^{\circ} 11$ de las sentencias 2088-2011, 2089-2011, 2090-2011.

35 Tribunal Constitucional. 5 de octubre de 2011, Rol 2088-2011. "Requerimiento de inaplicabilidad por inconstitucionalidad presentado por Rubén Pedro Molina Oyarzo respecto de los artículos segundo y tercero del Decreto Ley $N^{\circ} 3.500$ de 1980". Considerando 12. 
Es interesante el razonamiento del Tribunal Constitucional en estos fallos porque pareciera haber una "inconsistencia". En efecto, si el Tribunal Constitucional declara inadmisible la segunda presentación por no haberse invocado la gestión pendiente en la que recaería la inaplicabilidad, no puede -luego- señalar que el requerimiento también es inadmisible por haberse presentado un requerimiento anterior, en la misma gestión pendiente. La pregunta que salta a la vista es ¿cuál gestión pendiente, si la segunda presentación la declaró inadmisible por no haber invocado una gestión pendiente? ¿Basta con que el requerimiento sea presentado por el mismo requirente sobre las mismas normas, para presumir que se presenta desde la misma gestión pendiente?

En un cuarto caso ${ }^{36}$, el abogado presentó dos requerimientos iguales, y en la misma fecha ${ }^{37}$. Ambos requerimientos se tuvieron por no presentados por no haberse adjuntado el certificado de gestión pendiente. Luego con fecha 16 de octubre se vuelve a presentar un requerimiento de inaplicabilidad, en la misma gestión pendiente de los dos anteriores. Ese requerimiento fue declarado inadmisible por no encontrarse razonablemente fundado, y además porque habían sido presentados esos dos requerimientos que no fueron acogidos a trámite ${ }^{38}$. El razonamiento es del siguiente tenor:

"Por otra parte, debe tenerse presente que los actores del presente proceso constitucional formularon con anterioridad los requerimientos Roles $N^{\circ}$ s 2309 y 2310, los cuales no fueron acogidos a tramitación”.

En este caso el razonamiento del Tribunal llama la atención, debido a que, si bien los requerimientos son iguales, los juicios que sirven de gestión pendiente no son los mismos. El Tribunal, con la sola equivalencia de las partes y del requerimiento, lo declaró inadmisible. Así, el razonamiento del Tribunal Constitucional, no parece dentro del "contexto de lo que exige un proceso racional y justo".

En el quinto caso $^{39}$ el requerimiento es declarado inadmisible "derechamente" por varias razones. En primer término, por no encontrarse razonablemente fundado. Además, por no haberse presentado el certificado

\footnotetext{
36 Tribunal Constitucional. 13 de noviembre de 2012. Rol 2331-2012. "Requerimiento de inaplicabilidad por inconstitucionalidad presentado por Leonidas Alejandro Contin $\mathrm{Na-}$ ranjo y Antonio Contin González, respecto del inciso segundo del artículo 277 del Código Procesal Penal, en los autos criminales, de que conoce el Cuarto Juzgado de Garantía de Santiago, bajo el RIT 7268-2009, RUC 0800423642-5”.

37 Me refiero a los requerimientos presentados el 14 de septiembre de 2012 y, cuyos roles son 2309-2012 y 2310-2012.

38 Véase el considerando $8^{\circ}$ de la Sentencia del Tribunal Constitucional de fecha 13 de noviembre de 2012.

39 TC. Rol 2247-2012.
} 
de gestión pendiente en la fecha solicitada. Por último, por haberse presentado anteriormente un requerimiento de las mismas características ${ }^{40}$.

Ahora procederé a describir el único caso en que el TC no objeto -expresamente- el requerimiento por haberse presentado anteriormente uno similar.

Existe un caso ${ }^{41}$ en que se presentó por segunda vez un requerimiento de inaplicabilidad y este fue declarado inadmisible. La razón para esta declaración fue que el precepto no resultaba decisivo para la resolución del asunto ${ }^{42}$. Lo anterior, a pesar de que en los alegatos de admisibilidad el requerido alegó que no era posible presentar un segundo requerimiento en la misma gestión pendiente. El Tribunal en este caso no se pronunció acerca de los alegatos, y declaró inadmisible el segundo requerimiento por otra causal.

\section{Casos no declarados inadmisibles ${ }^{43}$}

Existen dos $\operatorname{casos}^{44}$ en los que, no obstante haberse presentado por segunda vez el requerimiento, este no se declaró inadmisible. En ambos el Tribunal Constitucional no se refirió en sus resoluciones a la primera presentación. Sin embargo estos casos difieren en que en uno se discutió el fondo del asunto y en el otro los requirentes se desistieron justo antes que se discutiera el fondo.

40 Tribunal Constitucional. 8 de mayo de 2012. Rol 2218-2012. "Requerimiento de inaplicabilidad por inconstitucionalidad presentado por Gastón Holzapfel Gross respecto "de la forma como se han aplicado" las disposiciones que indica, en los autos sobre reforma procesal penal RUC 0410014473-0, RIT 1930-2004, del Juzgado de Garantía de Valdivia”. Tribunal Constitucional. 5 julio de 2011. Rol 2013-2011. "Requerimiento de inaplicabilidad por inconstitucionalidad presentado por René Varas González respecto del artículo 24 del Decreto con Fuerza de Ley $N^{\circ}$ 2, de 1998, del Ministerio de Educación, en los autos Rol N $\mathrm{N}^{\circ}$ 71-2011 sobre recurso de protección interpuesto ante la Corte de Apelaciones de San Miguel en contra del Centro Educacional Principado de Asturias, del que conoce actualmente la Corte Suprema bajo el Rol N 4956-2011”.

42 El primer requerimiento había sido tenido "por no presentado" por no haberse acompañado dentro de tercero día el certificado de gestión pendiente.

43 Estos casos son sumamente difíciles de detectar, porque se debe tener un listado con una serie de antecedentes para verificar la triple identidad. Por esta razón, es posible que existan más casos que no fueron detectados.

44 Tribunal Constitucional. 3 de noviembre de 2011. Rol 1881-2011. "Requerimiento de inaplicabilidad por inconstitucionalidad presentado por la Corte de Apelaciones de Santiago respecto del artículo 102 del Código Civil, en los autos Rol No 6787-2010, sobre recurso de protección interpuesto ante la Corte de Apelaciones de Santiago en contra de Oficial de Registro Civil". El segundo caso es la sentencia del Tribunal Constitucional de fecha 5 de julio de 2011, Rol 1759- 2011. "Requerimiento de inaplicabilidad por inconstitucionalidad presentado por Alejandro Jordán Herrera, respecto del artículo 33 a) bis, letra C, No5, de la Ley No 18.933 o artículo 189, letra C, No5, del DFL No1, del año 2005, del Ministerio de Salud, en los autos Rol No 438-2010 sobre recurso de protección interpuesto ante la Corte de Apelaciones de Santiago en contra de Isapre Colmena Golden Cross". 
En uno de esos casos ${ }^{45}$ el Tribunal Constitucional no se refirió al primer requerimiento y una de las razones que pareciera explicarlo es que el sujeto activo, es decir, quien presentó el requerimiento era distinto. En efecto, la primera presentación fue realizada por uno de los recurrentes en el recurso de protección que sirvió de gestión pendiente para el asunto. El segundo requerimiento fue presentado por la Corte de Apelaciones de Santiago, Tribunal que debía resolver el recurso.

En el otro caso ${ }^{46}$, si bien no se alcanzó a resolver el fondo del asunto porque los requirentes se desistieron, el Tribunal tampoco se refirió al primer requerimiento, a pesar de que se presentó en la misma gestión pendiente y por el mismo requirente. Incluso el requerido en un escrito de "se tenga presente" señaló este elemento como causal para la declaración de inadmisibilidad. La razón que pareciera explicar por qué el Tribunal no lo declaró inadmisible es porque en la segunda presentación se impugnó otra norma, la norma que el Tribunal señaló en el primer fallo que debió ser impugnada, es decir, el requirente subsanó el error y volvió a presentar el requerimiento. Esto pareciera confirmar la tesis del Ministro Fernández en el Fallo Rol 1442-2011.

\section{4) Conclusiones}

Como se ha mostrado en el presente trabajo, el Tribunal Constitucional, en la mayoría de los $\operatorname{casos}^{47}$, ha declarado inadmisible los requerimientos de inaplicabilidad presentados por segunda vez en la misma gestión pendiente. Esto, a pesar de lo que parece indicar la LOCTC y lo que el profesor Tavolari indica. La LOCTC prohíbe derechamente la presentación de un requerimiento de inaplicabilidad nuevamente cuando ya ha sido resuelto un requerimiento sobre el mismo vicio y en la misma gestión pendiente. Esto no sucede en el caso en que el primer requerimiento sea declarado inadmisible (porque el requerimiento no ha sido resuelto), por lo que es dable concluir que dicha prohibición no existe. El profesor Tavolari y el profesor Nogueira siguen esa opinión. No es posible captar la razón que justifica que el Tribunal Constitucional declare inadmisible el requerimiento, presentado por segunda vez en la misma gestión pendiente, invocando un vicio diverso. Sobre este punto existen dos tendencias. La primera

TC, Rol 1881-2010.

TC, Rol 1759- 2011. La primera presentación tiene el Rol 1739.

Queda la duda en el caso en donde el segundo requerimiento es presentado en la misma gestión pendiente, invocándose igual vulneración constitucional, pero en el primer caso se requirió por una parte, y en el segundo por el juez de la gestión. Otro caso hipotético en el que quedarían dudas sería que se presentaran los mismos requerimientos, pero en una causa acumulada, de manera que cada parte en cada juicio de la gestión acumulada presentara su requerimiento. 
señala que el segundo requerimiento debe ser declarado inadmisible a pesar de que el requirente lo haya enmendado e invoque un vicio diverso y la segunda declara el segundo requerimiento admisible, sin referirse a esa causal. En este punto el Tribunal debiera tomar una decisión consistente y optar por una de las tendencias, a pesar de que la primera no tenga sustento normativo.

Además queda la duda del por qué en un caso el segundo requerimiento fue declarado inadmisible por otra causal, sin hacer mención a la primera presentación. También queda la duda de si es posible que en la misma gestión pendiente, otra parte presente un nuevo requerimiento. Pareciera ser que el Tribunal Constitucional pretende crear un nuevo filtro para seleccionar los requerimientos de inaplicabilidad en los que decidirá el fondo del asunto, pero si ese es el plan debe ser consistente.

\section{5) Bibliografía}

- Casarino, Mario (2005) Manual de derecho procesal. Santiago de Chile: Editorial Jurídica de Chile.

- Cea, José Luis (2010). "Nueva Ley Orgánica del Tribunal Constitucional: Una síntesis compleja”. Revista Chilena de Derecho, Vol. 37, No 1.

- Colombo, Juan (2008) Los actos procesales. Santiago de Chile: Editorial Jurídica de Chile.

- Correa, Jorge (2011) Inaplicabilidad por inconstitucionalidad en la jurisprudencial del Tribunal Constitucional. Santiago de Chile: Abeledo Perrot.

- Navarro, Enrique (2010). "Acción de inaplicabilidad por inconstitucionalidad. Notas sobre la evolución histórica en Chile”. Revista Actualidad Juridica, No 22.

- Nogueira, Humberto (2005). "El control represivo concreto y abstracto de inconstitucionalidad”. En Zúñiga, Francisco (editor): Reforma Constitucional. Santiago de Chile: LexisNexis.

- Otero, Miguel (2000) Derecho Procesal civil. Modificaciones a la legislación (1988-2000). Santiago de Chile: Editorial Jurídica de Chile.

- Pica, Rodrigo (2010). "El procedimiento correspondiente al proceso de inaplicabilidad por inconstitucionalidad en la Ley Orgánica Constitucional del Tribunal Constitucional”. En Nogueira, Humberto (editor): Temas de derecho procesal constitucional: Reflexiones juridicas sobre competencias del Tribunal Constitucional y la nueva LOC del Tribunal Constitucional. Santiago de Chile: Librotecnia. 
TAvolari, Raúl (2010). "La cosa juzgada en el control de constitucionalidad”. Revista de derecho público, Vol. 72.

\section{JuRisprudenCia CitAdA}

- Tribunal Constitucional. Rol No 1036-2008. "Requerimiento de inaplicabilidad por inconstitucionalidad de Mario Alberto Cortés Alarcón respecto del DFL. No 3, de 1.997 y en subsidio, el artículo 103 y siguientes, especialmente el artículo 138, antes 135 de la Ley General de Bancos”.

- Tribunal Constitucional. Rol N 1265-2008. "Requerimiento de inaplicabilidad por inconstitucionalidad de Sonia Angélica Cifuentes Olea respecto del artículo 38 ter de la Ley No 18.933”.

- Tribunal Constitucional. Rol No 1282-2008. "Requerimiento de inaplicabilidad de Sonia Angélica Cifuentes Olea respecto del artículo 38 ter de la Ley No 18.933”.

- Tribunal Constitucional. Rol N 1311-2009. "Requerimiento de inaplicabilidad por inconstitucionalidad de Mario Alberto Cortés Alarcón respecto de los artículos 103 y siguientes, en especial del actual 138, antes 135, del Decreto con Fuerza de Ley No 3, del año 1997, que fija el texto refundido y sistematizado de la Ley General de Bancos".

- Tribunal Constitucional. Rol No 1420-2009. "Requerimiento de inaplicabilidad por inconstitucionalidad de Isapre Más Vida S.A., respecto del artículo transitorio de la Ley 20.317”.

- Tribunal Constitucional. Rol No 1442-2009. "Requerimiento de inaplicabilidad por inconstitucionalidad de Isapre Más Vida S.A., respecto del artículo transitorio de la Ley 20.317".

- Tribunal Constitucional. Rol No 1581-2010. "Requerimiento de inaplicabilidad por inconstitucionalidad de Pedro Hernán Lonch Contreras, respecto del No 2 de la Ley No 20.015 del Ministerio de Salud".

- Tribunal Constitucional. Rol N 1590-2010. "Requerimiento de inaplicabilidad por inconstitucionalidad respecto del artículo 2, No 2, de la ley 20.015 de 17 de mayo de 2005, del Ministerio de Salud, que "modifica la ley No 18.933, sobre Isapres"”.

- Tribunal Constitucional. Rol No 1671-2010. "Requerimiento de inaplicabilidad por inconstitucionalidad de Pedro Hernán Lonch Contreras respecto de los artículos 1.545 del Código Civil, 38 de la Ley No 18.933 y 20 de la Ley No 20.015”.

- Tribunal Constitucional. Rol No 1672-2010. "Requerimiento de inaplicabilidad por inconstitucionalidad de Rogelio Arnaldo Vargas 
Nilo respecto de los artículos 1.545 del Código Civil, 38 de la Ley No 18.933 y $2^{\circ}$ de la Ley No $20.015^{\prime}$.

- Tribunal Constitucional. Rol No 1678-2010. "Requerimiento de inaplicabilidad por inconstitucionalidad de Alberto Michel Haddad Valech, respecto del artículo 212 del Código Civil”.

- Tribunal Constitucional. Rol No 1739-2010. "Acción de inaplicabilidad por inconstitucionalidad de Alejandro Jordán Herrera respecto del artículo 2 de la Ley No 20.015".

- Tribunal Constitucional. Rol No 1759-2010. "Acción de inaplicabilidad por inconstitucionalidad de Alejandro Jordán Herrera respecto del artículo 33 a) bis, letra C, No5, de la Ley No 18.933 o artículo 189, letra C, No5, del DFL No1, del ańo 2005, del Ministerio de Salud".

- Tribunal Constitucional. Rol No 1834-2010. "Requerimiento de inaplicabilidad por inconstitucionalidad de Alberto Michel Haddad Valech respecto del artículo 212 del Código Civil”.

- Tribunal Constitucional. Rol No 1840-2010. "Requerimiento de inaplicabilidad respecto al artículo 102 del Código civil”.

- Tribunal Constitucional. Rol No 1881-2010. "Requerimiento de inaplicabilidad por inconstitucionalidad presentado por la Corte de Apelaciones de Santiago respecto del artículo 102 del Código Civil".

- Tribunal Constitucional. Rol No 1936-2011. "Requerimiento de inaplicabilidad por inconstitucionalidad presentado por Guido Ernesto Andrade Pillancare respecto de los artículos segundo y tercero del Decreto Ley No 3.500".

- Tribunal Constitucional. Rol No 1937-2011. "Requerimiento de inaplicabilidad por inconstitucionalidad presentado por Rubén Pedro Molina Oyarzo respecto de los artículos segundo y tercero del Decreto Ley No 3.500".

- Tribunal Constitucional. Rol No 1938-2011. "Requerimiento de inaplicabilidad por inconstitucionalidad presentado por José Ignacio Velásquez Alarcón respecto de los artículos segundo y tercero del Decreto Ley No 3.500".

- Tribunal Constitucional. Rol No 1977-2011. "Requerimiento de inaplicabilidad por inconstitucionalidad presentado por Clara Inés Weber Chávez y otros respecto del artículo 24 del Decreto con Fuerza de Ley $\mathrm{N}^{\circ} 2$, de 1998, del Ministerio de Educación, sobre subvención del Estado a establecimientos educacionales".

- Tribunal Constitucional. Rol No 2013-2011. "Requerimiento de inaplicabilidad por inconstitucionalidad presentado por Clara Inés Weber Chávez y otros respecto del artículo 24 del Decreto con Fuerza de Ley $\mathrm{N}^{\circ}$ 2, de 1998, del Ministerio de Educación, sobre subvención del Estado a establecimientos educacionales". 
- Tribunal Constitucional. Rol No 2088-2011. "Requerimiento de inaplicabilidad por inconstitucionalidad presentado por Rubén Pedro Molina Oyarzo respecto de los artículos segundo y tercero del Decreto Ley No 3.500 de 1980".

- Tribunal Constitucional. Rol No 2089-2011. "Requerimiento de inaplicabilidad por inconstitucionalidad presentado por María Elvira Astaburuaga Castañeda respecto de los artículos segundo y tercero del Decreto Ley No 3.500 de 1980".

- Tribunal Constitucional. Rol No 2090- 2011. "Requerimiento de inaplicabilidad por inconstitucionalidad presentado por Guido Ernesto Andrade Pillancare respecto de los artículos segundo y tercero del Decreto Ley No 3.500 de 1980".

- Tribunal Constitucional. Rol No 2218-2012. "Requerimiento de inaplicabilidad por inconstitucionalidad presentado por Gastón Holzapfel Gross, respecto "de la forma como se han aplicado" las disposiciones que indica”.

- Tribunal Constitucional. Rol No 2247-2012. "Requerimiento de inaplicabilidad por inconstitucionalidad presentado por Gastón Holzapfel Gross respecto de los artículos 3, 229, 334, 342, letra f), 370 y 483 del Código Procesal Penal”.

- Tribunal Constitucional. Rol No 2309-2012. "Requerimiento de inaplicabilidad por inconstitucionalidad presentado por Leonidas Alejandro Contin Naranjo respecto del inciso segundo, del artículo 277 del Código Procesal Penal”.

- Tribunal Constitucional. Rol No 2310-2012. "Requerimiento de inaplicabilidad por inconstitucionalidad presentado por Antonio Contin González respecto del inciso segundo del artículo 277 del Código Procesal Penal".

- Tribunal Constitucional. Rol No 2331-2012. "Requerimiento de inaplicabilidad por inconstitucionalidad presentado por Leonidas Alejandro Contin Naranjo y Antonio Contin González, respecto del inciso segundo del artículo 277 del Código Procesal Penal”. 